\title{
Vietnam - Regionale Disparitäten in einem Entwicklungsland mit dynamischer Wirtschaftsentwicklung
}

\section{Khanh Hung Duong, Basel}

\section{Einführung}

Vietnam begann 1986 nach Jahren der rückläufigen Entwicklung und einer schweren ökonomischen Krise einen Transformationsprozess. Bekannt als Doi Moi $(=$ Erneuerung) bedeutete er die Abkehr von einer zentral gelenkten Planwirtschaft hin zu einer Marktwirtschaft sozialistischer Prägung. Nach enormen Anpassungsproblemen gegen Ende der Dekade, die Vietnam in die grösste Hungersnot seit 1945 stürzte, avancierte die Nation Angaben der WeltBank (1999a: 38) zufolge in den 1990er Jahren zu einer der zehn am schnellsten wachsenden Ökonomien der Erde. Zwischen 1990 und 2000 wuchs das Bruttoinlandprodukt (BIP) im Durchschnitt jährlich um 7,4\%. Die Inflationsrate - Mitte der 1980er Jahre noch im Hyperinflationsbereich - sank auf unter $10 \%$ p.a., Aussenhandel und ausländische Direktinvestitionen nahmen beträchtlich zu. Das ProKopf-Einkommen konnte im gleichen Zeitraum von 97 US\$ auf 400 US\$ gesteigert werden. Wenngleich Vietnam nach wie vor zu den ärmsten Ländern der Erde zählt, so sind die Erfolge der vergangenen 15 Jahre beachtlich. Vietnam ist in zunehmendem Masse Gegenstand wissenschaftlicher Abhandlungen (MARR 2002; WaIBel 2003; DuONG 2003), und viele Kommentatoren sehen das Land bereits als nächsten asiatischen «Tiger» (u.a. Nuscheler 1999: 11; WeSSEL 1998: 14).

So zutreffend solche Einschätzungen auf gesamtnationaler Ebene auch sein mögen - dies belegen die Daten zur gesamtwirtschaftlichen Entwicklung des Landes (Tab. 1) -, sagen sie doch nichts darüber aus, ob und in welchem Ausmass die Einzelregionen des Landes von diesem gesamtnationalen Aufschwung profitiert haben. Der vorliegende Beitrag beschäftigt sich mit dem regionalen Aspekt der Entwicklungsdynamik in den 1990er Jahren.

\section{Fragestellung und methodisches Vorgehen}

Bronger \& Strelow (1996: 20) folgend wird in diesem Beitrag Entwicklung verstanden als die Partizipation eines immer grösser werdenden Anteils der Bevölkerung eines Landes an dem wachsenden materiellen, sozialen und kulturellen Wohlstand. Diese Auffassung impliziert einerseits, dass Entwicklung ohne
Wachstum nicht denkbar ist. Anderseits hebt sie die Bedeutung verteilungspolitischer Aspekte hervor, um «Wachstum ohne Entwicklung» auszuschliessen.

Erst eine raumdifferenzierende Betrachtung von Entwicklungsprozessen ermöglicht es, Aussagen über die Partizipation der Bevölkerung am wachsenden gesamtgesellschaftlichen Wohlstand zu treffen. Dies hat als Entscheidungsgrundlage für eine regionale Entwicklungsplanung und -politik in Vietnam eine besondere Bedeutung: Der erste grössere Aufstand der Landbevölkerung 1997 in der Provinz Thai Binh (Rotes Fluss Delta) gegen Regierung und Partei (BäNZIGER 2000) spiegelt die Gefahr von gesellschaftspolitischen Konflikten wider, sollte die Kluft zwischen Stadt und Land oder Arm und Reich - kurz: das regionale Entwicklungsgefälle - grösser werden.

$\mathrm{Zu}$ fragen ist demnach: Wie ist die Entwicklung regional verlaufen? Haben alle oder hat zumindest ein Grossteil der Regionen und Menschen von dieser positiven gesamtnationalen Entwicklung profitieren können? Oder fand vielmehr eine punktuelle Entwicklung statt, die sich in einer ausgesprochenen Primatstellung der Metropolen Ho Chi Minh City (HCMC) und Ha Noi ausdrückt? Ferner wirft die Notwendigkeit einer ausgewogenen Regionalpolitik die Frage nach der Rolle des vietnamesischen Staates im Entwicklungsprozess auf. Mit welchen Mitteln versucht er räumliche Disparitäten abzubauen oder zumindest zu mildern?

Anhand ausgewählter Indikatoren aus verschiedenen Lebensbereichen wird das regionale Entwicklungsgefälle auf Grundlage der 61 Provinzen Vietnams (Karte 1) analysiert. Um den Entwicklungsverlauf der jeweiligen Provinzen aufzuzeigen, werden die verwendeten Indikatoren nicht in ihrer absoluten, sondern in ihrer realen Bedeutung ausgedrückt, so dass die in Fläche und Grösse heterogenen Raumeinheiten gemäss ihrer tatsächlichen Einwohnerstärke berücksichtigt werden. Für die Indexberechnung der Einzelprovinzen wird der Pro-Kopf-Anteil des jeweiligen Indikators real, bezogen auf den nationalen Durchschnitt (Vietnam $=100$ ) wiedergegeben. Um Aussagen über Ausmass und Dynamik des regionalen Entwicklungsgefälles abzuleiten, wird zunächst die Variationsbreite ermittelt, die das Verhältnis zwischen der «reichsten" und «ärmsten" Provinz ausdrückt. Da auf diese Weise nur die beiden möglicherweise atypischen Extremwerte berücksichtigt werden, wird 


\begin{tabular}{|c|c|c|c|c|c|c|c|c|c|c|c|c|}
\hline \multicolumn{2}{|c|}{ Indikator } & 1990 & 1991 & 1992 & 1993 & 1994 & 1995 & 1996 & 1997 & 1998 & 1999 & 2000 \\
\hline \multirow{2}{*}{$\begin{array}{l}\text { Bruttoinlandprodukt } \\
\text { (BIP) }\end{array}$} & pro Kopf in USs ${ }^{1}$ & 97 & 130 & 145 & 186 & 221 & 290 & 339 & 363 & 361 & 376 & 400 \\
\hline & \begin{tabular}{|l|} 
Veränderung zum \\
Vorjahr in $\%^{2}$ \\
\end{tabular} & 5,1 & 5,8 & 8,7 & 8,1 & 8,8 & 9,5 & 9,3 & 8,2 & 5,8 & 4,8 & 6,8 \\
\hline Inflationsrate ${ }^{1}$ & in $\%$ & 67,5 & 67,6 & 17,5 & 5,2 & 14,4 & 12,7 & 4,5 & 3,6 & 9,2 & 0,1 & $-0,6$ \\
\hline Staatseinnahmen ${ }^{4}$ & in $\%$ des BIP & 14,7 & 13,5 & 19,0 & 22,5 & 24,7 & 23,9 & 22,9 & 21,1 & 19,0 & 17,8 & $20,3^{2}$ \\
\hline Staatsausgaben $^{4}$ & in $\%$ des BIP & 20.5 & 15,2 & 20,7 & 27,0 & 25,8 & 24,0 & 23,1 & 22,6 & 20,1 & 19,1 & $22,2^{2}$ \\
\hline Haushaltsbilanz & in $\%$ des BIP & $-5,8$ & $-1,7$ & $-1,7$ & $-4,5$ & $-1,1$ & $-0,1$ & $-0,2$ & $-1,5$ & $-1,1$ & $-1,3$ & $-1,8$ \\
\hline Exporte $^{6}$ & in Mio. US\$ & 2.404 & 2.087 & 2.581 & 2.985 & 4.054 & 5.449 & 7.256 & 9.185 & 9.361 & 11.523 & $14.500^{2}$ \\
\hline Importe $^{6}$ & in Mio. US\$ & 2.752 & 2.338 & 2.541 & 3.924 & 5.826 & 8.155 & 11.144 & 11.592 & 11.527 & 11.636 & $15.600^{2}$ \\
\hline $\begin{array}{l}\begin{array}{l}\text { Ausländische Direkt- } \\
\text { investitionen }\end{array}{ }^{2} \\
\end{array}$ & in Mio. US\$ & 839 & 1.322 & 2.165 & 2.900 & 3.766 & 6.531 & 8.497 & 4.649 & 3.897 & 1.568 & 2.012 \\
\hline Reisproduktion $^{2,7}$ & in Tsd. $t$ & 19.225 & 19.622 & 21.590 & 22.837 & 23.528 & 24.964 & 26.397 & 27.524 & 29.146 & 31.394 & 32.554 \\
\hline $\begin{array}{l}\text { Reisproduktion pro } \\
\text { Kopf }^{2,7}\end{array}$ & in $\mathrm{kg}$ & 294,2 & 295,3 & 319,4 & 332,1 & 336,4 & 350,8 & 364,6 & 373,5 & 388,7 & 411,3 & 419 \\
\hline Reisexport ${ }^{2,7}$ & in Tsd. $t$ & 1.624 & 1.033 & 1.946 & 1.722 & 1.983 & 1.988 & 3.003 & 3.575 & 3.730 & 4.508 & 3.500 \\
\hline
\end{tabular}

Tab. 1: Ausgewählte Indikatoren zur gesamtwirtschaftlichen Entwicklung Vietnams 1990-2000

Selected indicators of overall development in Vietnam between 1990 and 2000

Choix d'indicateurs sur le développement économique général du Vietnam, de 1990 à 2000

Quellen: 'United Nations Development Programme 2001b; 'General. Statistical Office 2001: 70: 'Wolff 1997: 17; ${ }^{4}$ Weltbank 1999b (Statistical Appendix); ${ }^{5}$ Weltbank 1994: 125 ff.; ${ }^{\circ}$ Asian Development Bank 2000: 382 ff.; ${ }^{7}$ General Statistical Office 1997, 2000c; eigene Berechnungen

zusätzlich der gewogene Variationskoeffizient $(\mathrm{Vg})$ ermittelt. Mit diesem Ansatz wird die Abweichung der regionalen Pro-Kopf-Werte vom durchschnittlichen Pro-Kopf-Wert des Landes errechnet, wobei die Abweichung jeweils mit der regionalen Bevölkerungsstärke gewichtet wird. Je grösser der Wert für $\mathrm{Vg}(0=$ völlige Gleichverteilung), desto grösser ist die Streuung des jeweiligen Indikators um den nationalen Durchschnitt und desto grösser ist das Ausmass der Ungleichverteilung.

\section{Das regionale Entwicklungsgefälle: Ausgewählte Untersuchungsergebnisse}

\subsection{Wirtschaft}

Die Analyse der Raumstruktur in Vietnam muss vor dem Hintergrund der jüngeren Wirtschaftsgeschichte des Landes betrachtet werden. Das heutige bipolare Raummuster entwickelte sich bereits seit der zweiten Hälfte des 19. Jahrhunderts unter französischer Kolonialherrschaft. Die damals aufgebaute koloniale Exportökonomie konzentrierte sich von Beginn an auf die Entwicklungsdreiecke Ha Noi-Hai Phong-Quang Ninh im nördlichen und HCMC-Dong Nai-Vung Tau im südlichen Teil Vietnams (LE BA Thao 1997: 260). Begünstigt durch den unter französischem Einfluss eingeleiteten Industrialisierungsprozess konnten $\mathrm{Ha}$ Noi und Sai Gon (heute HCMC) zusätzlich ihre dominierende Stellung abwechselnd als Verwaltungszentren von ganz Indochina ausbauen (vgl. MARR 2002). Die anhaltende Bevorzugung der beiden Zentren im Zusammenhang mit den früh entstandenen Standortnachteilen in den restlichen Landesteilen zeigt ihre Auswirkungen bis in die heutige Zeit hinein: Den beiden Industrie- und Wirtschaftszentren stehen auf der anderen Seite bis auf wenige Ausnahmen nahezu "weisse Flecken" auf der gewerblich-industriellen Landkarte gegenüber. Bestätigt wird dieser Entwicklungsverlauf durch die Analyse-Ergebnisse der Indikatoren BIP. Ausländische Direktinvestitionen (FDI) und Industrielle Bruttoproduktion:

- BIP: Bei nahezu zwei Dritteln der Provinzen ist gemessen am Landesdurchschnitt - eine rückläufige Entwicklung gegenüber 199() zu konstatieren. Waren es zu Beginn noch 16 Provinzen. die das Landes- 
mittel erzielen konnten, reduzierte sich ihre Zahl 1998 auf 13. Während die Variationsbreite zwischen der «reichsten» und der «ärmsten» Provinz - bis 1994 ansteigend, dann wieder fallend - nahezu unverändert geblieben ist, weist der Indexwert für sechs der acht Regionen eine abnehmende Tendenz auf. In Karte 2 wird das ganze Ausmass des regionalen Entwicklungsgefälles offenkundig.

- FDI (registriertes Kapital): Wie bei keinem anderen Untersuchungsindikator kann in diesem Bereich von einer punktuellen Entwicklung gesprochen werden, von der nahezu ausschliesslich die beiden Kernzentren $\mathrm{HCMC} / \mathrm{Ba}$ Ria-Vung Tau und $\mathrm{Ha}$ Noi sowie ihre Umlandprovinzen profitiert haben. Der Grossteil des Landes lag hingegen - zu Beginn wie auch zum Ende des Untersuchungszeitraums - abseits der Investitionsströme (Karte 3). Das eklatante Gefälle schlägt sich in dem Verhältnis zwischen der «reichsten» und «ärmsten» Provinz (6.650 [!] : 1) nieder.

- Industrielle Bruttoproduktion: Zwar konnten die regionalen Disparitäten in diesem Bereich deutlich abgebaut werden, die Variationsbreite beträgt 2000 aber immer noch 277 : 1. Insgesamt konnte im Verlauf der vergangenen Dekade neben der Südostregion mit HCMC und Ba Ria-Vung Tau, dem Zentrum der Erdöl- und Erdgasindustrie, keine andere Region des Landes überdurchschnittliche Indexwerte (über 100) vorweisen. Entsprechend deutlich kommen die räumlichen Unterschiede in Karte 4 zum Ausdruck.

Auch mit dem Ansatz des gewogenen Variationskoeffizienten $(\mathrm{Vg})$ verfestigen sich die bisher festgestellten Befunde. Mit einem Wert von annähernd 0,8 in 1998 bleibt das Entwicklungsgefälle beim BIP weiterhin auf einem hohen Niveau. Zum Vergleich: Der Vg-Wert für das BIP lag für Thailand bei 1,018 (1991), für die Philippinen bei 0,601 (1993) und für Südkorea bei 0,166 (1992) (Bronger \& Strelow 1996: 208). Noch deutlicher werden die wirtschaftlichen Disparitäten in den Bereichen Industrielle Bruttoproduktion und FDI. Für den gesamten Untersuchungszeitraum weisen hier die Variationskoeffizienten Werte von über 1,6 auf, im Falle der FDI von über 2,6 und 4,2 für die Jahre 1999 und 2000 (Tab. 2).

\subsection{Bildung}

Im höheren (tertiären) Bildungsbereich bestätigt ein Blick auf die räumliche Verteilung der Universitätsund Collegestudierenden das enorme Ausmass der Disparitäten. Die Entwicklung auf nationaler Ebene vernahm zwar einen erfreulichen Verlauf - die Zahl der eingeschriebenen Studierenden stieg im Zeitraum 1990-99 von 93 Tsd. auf 412 Tsd. an -, mit der regionalen Aufschlüsselung der Daten offenbaren sich aber die Entwicklungsunterschiede in diesem Lebensbereich.
Das überragende Zentrum ist die Hauptstadt $\mathrm{Ha}$ Noi, in der die erste Universität Vietnams schon im 11. Jahrhundert gegründet wurde. Ihre bereits 1990 bestehende Dominanz konnte im Verlauf des Betrachtungszeitraums nicht abgebaut werden. 1999 lag die Studierendendichte in der Hauptstadt (55 Studierende pro Tsd. Einw.) noch immer beim fast 10fachen Landesdurchschnitt. Gemessen an den absoluten Studierendenzahlen kann allenfalls $\mathrm{HCMC}$ als einzige Provinz mit der Entwicklung in Ha Noi mithalten. An zehn Provinzen, besonders in den nördlichen Bergregionen, ging die Entwicklung gänzlich vorbei. Bis heute gibt es dort überhaupt keine Universitäts- und Collegestudierende. Die ungleiche Entwicklung auf regionaler Ebene belegen eindrücklich die ermittelten $\mathrm{Vg}$-Werte für die Jahre 1990 und 1999 (Tab. 2).

\subsection{Gesundheit}

Der Gesundheitsstatus in Vietnam ist gemessen am niedrigen Einkommensstandard des Landes bemerkenswert gut. Die zur Verfügung stehenden Krankenhausbetten und die Anzahl der Ärzte pro Kopf der Bevölkerung lassen im Vergleich mit den asiatischen Nachbarstaaten einen relativ hohen Versorgungsgrad erkennen (GerTler \& Litvack 1998: 236). Von der insgesamt guten Versorgung können die meisten Provinzen profitieren. Sowohl bei den Krankenhausbetten als auch bei den Ärzten erscheint die regionale Verteilung deutlich ausgeglichener als in den übrigen Lebensbereichen. Lediglich das Mekong Delta fällt gegenüber den anderen Regionen etwas ab (Abb. 1). Auf Provinzebene ist erfreulich, dass die wirtschaftlich rückständigen Gebiete in den nördlichen Bergprovinzen und im Zentralen Hochland gute, teilweise über dem nationalen Durchschnitt liegende Werte vorweisen können. Die ausgeprägte Dominanz der Provinz Da Nang gegenüber den restlichen Landesteilen konnte bis 2000 deutlich abgebaut werden, was sich in der geringeren Variationsbreite zwischen der «reichsten» und «ärmsten» Provinz widerspiegelt. Auch die Zeitreihenbetrachtung der Vg-Werte für beide Indikatoren macht das im Vergleich zu den anderen Lebensbereichen weniger stark ausgeprägte Entwicklungsgefälle deutlich (Tab. 2).

\subsection{Lebenssituation und Armut}

Nach Angaben der Weltbank (1999a: 3) konnte kaum ein anderes Land in den vergangenen zwei Dekaden einen so signifikanten Rückgang der Armut verzeichnen wie Vietnam. In welchem Ausmass die wirtschaftlichen Erfolge unter Doi Moi zur Verbesserung der Lebenssituation für die Bevölkerung beigetragen haben, zeigen die Ergebnisse der 1992/93 sowie 1997/98 durchgeführten Vietnam Living Standards Surveys. Danach lassen sich folgende Aussagen über die Veränderung der Armutssituation treffen: 


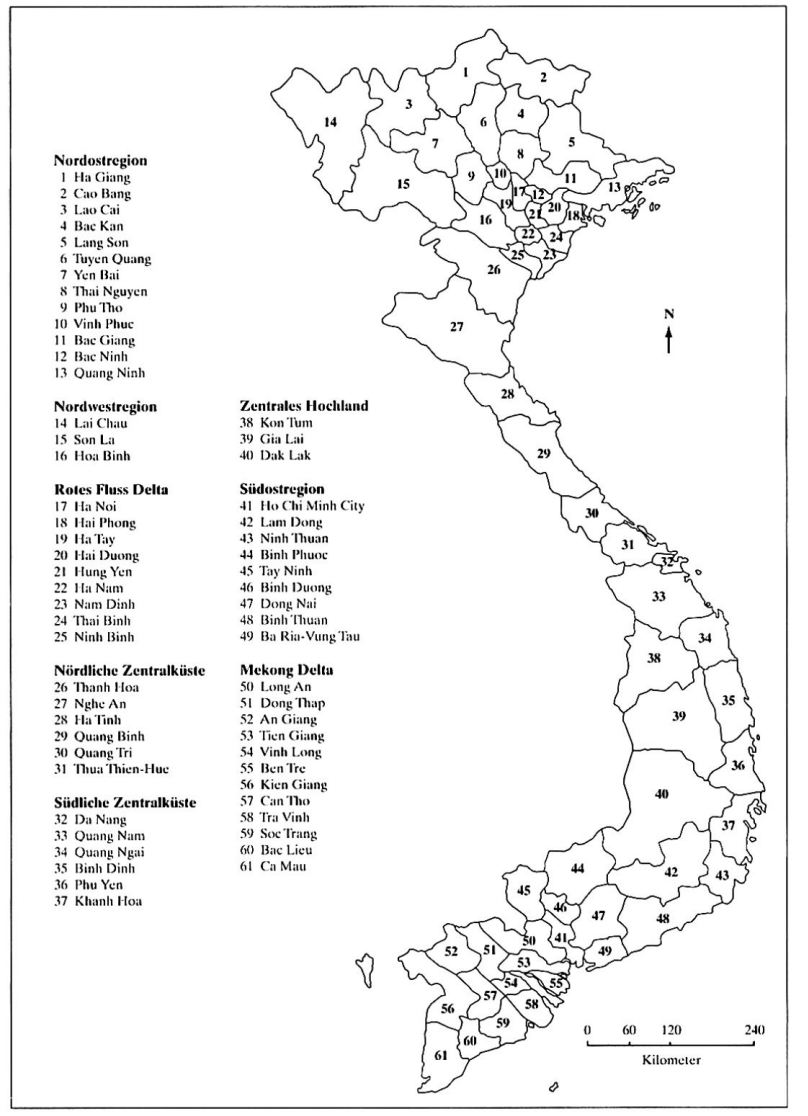

Karte 1: Politisch-administrative Gliederung Vietnams nach Regionen und Provinzen Political administrative division of Vietnam according to regions and provinces Structure politico-administrative du Vietnam, selon les régions et les provinces Quelle: Duong 2003; Kartengrundlage: UN 1999; Kartographie: K.H. Duong

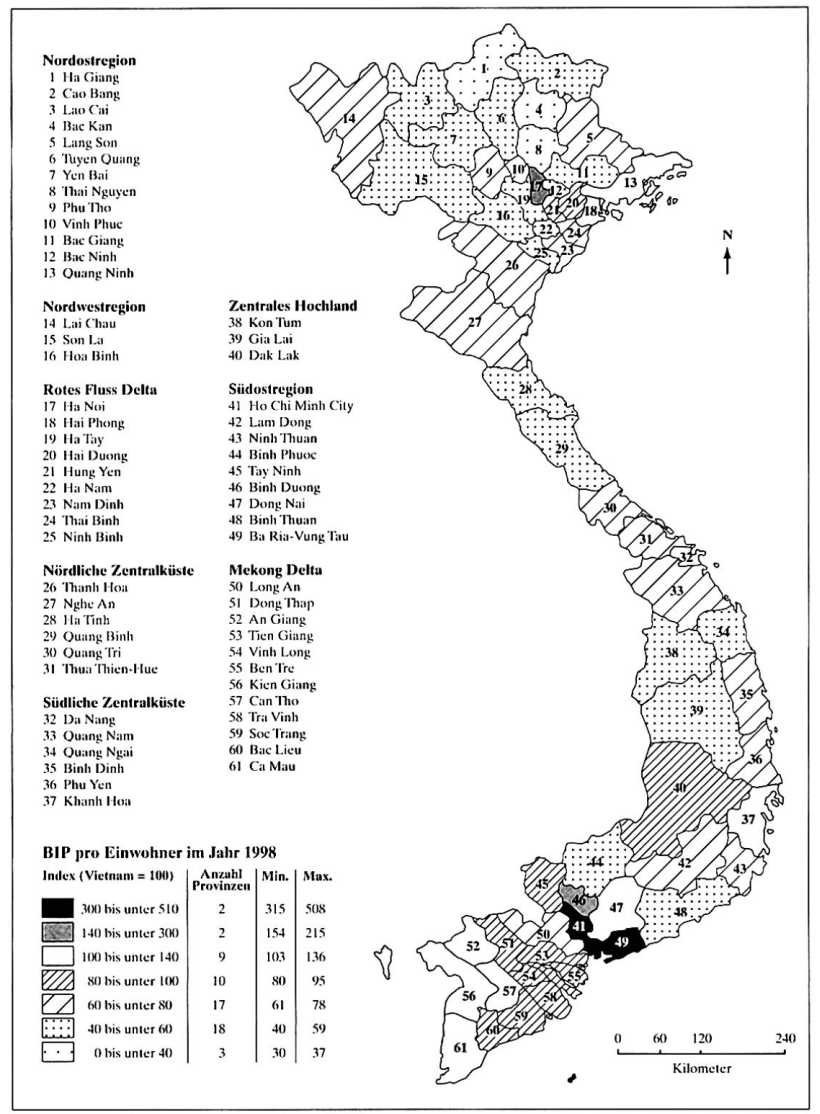

Karte 2: Bruttoinlandprodukt pro Kopf nach Provinzen 1998 Gross domestic product per person according to provinces 1998 Produit intérieur brut/habitant, selon les provinces (1998)

Quelle: Duong 2003; Kartengrundlage: UN 1999; Kartographie: K.H. DuoNG 


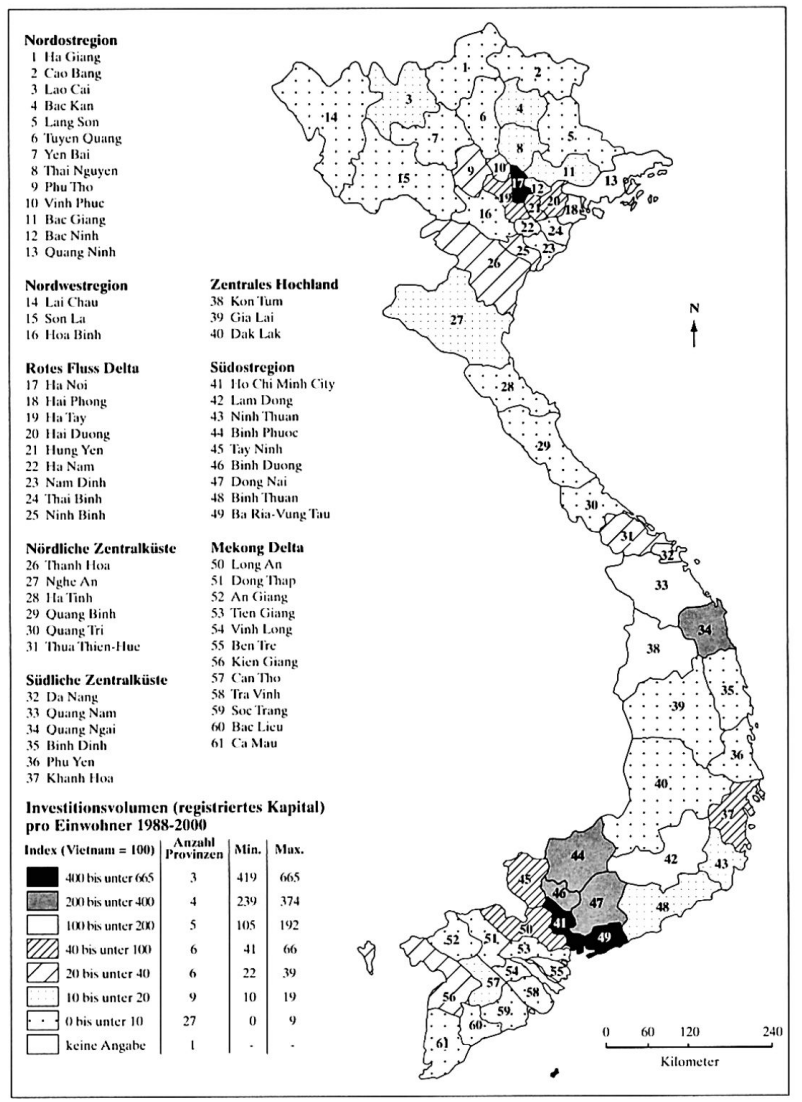

Karte 3: Ausländische Direktinvestitionen pro Kopf nach Provinzen 1988-2000 Foreign direct investment per person according to provinces 1988 to 2000

Investissements étrangers directs/habitant, selon les provinces (1988-2000)

Quelle: Duong 2003; Kartengrundlage: UN 1999; Kartographie: K.H. DuoNG

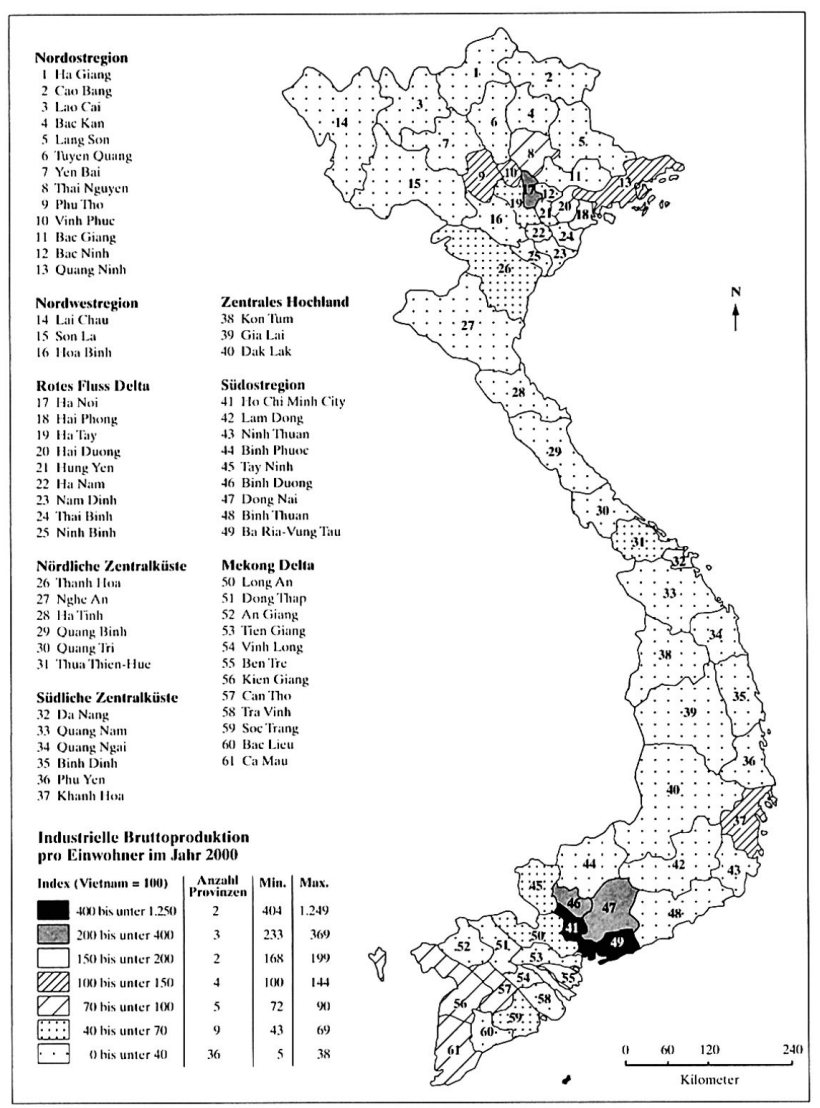

Karte 4: Industrielle Bruttoproduktion pro Kopf nach Provinzen 2000 Industrial gross production per person according to provinces 2000

Production industrielle brute/habitant, selon les provinces (2000) Quelle: Duong 2003; Kartengrundlage: UN 1999; Kartographie: K.H. DuonG 


\begin{tabular}{|l|c|c|c|c|c|c|c|c|c|c|c|}
\hline \multicolumn{1}{|c|}{ Indikator } & $\mathbf{1 9 9 0}$ & $\mathbf{1 9 9 1}$ & $\mathbf{1 9 9 2}$ & $\mathbf{1 9 9 3}$ & $\mathbf{1 9 9 4}$ & $\mathbf{1 9 9 5}$ & $\mathbf{1 9 9 6}$ & $\mathbf{1 9 9 7}$ & $\mathbf{1 9 9 8}$ & $\mathbf{1 9 9 9}$ & $\mathbf{2 0 0 0}$ \\
\hline $\begin{array}{l}\text { Bruttoinland - } \\
\text { produkt (BIP) }\end{array}$ & 0,5786 & 0,6242 & 0,7077 & 0,7489 & 0,7879 & 0,9054 & 0,8732 & 0,8392 & 0,7992 & - & - \\
\hline $\begin{array}{l}\text { Ausländische } \\
\text { Direktinvesti- } \\
\text { tionen (FDI) }\end{array}$ & - & - & - & - & - & - & 2,1327 & - & - & 2,6987 & 4,2242 \\
\cline { 2 - 12 } & \multicolumn{7}{|c|}{$1988-1995: 1,7926$} \\
\hline $\begin{array}{l}\text { Industrielle } \\
\text { Bruttoproduktion }\end{array}$ & - & 1,8094 & 2,0035 & 2,0121 & 1,9605 & 1,9015 & 1,6562 & 1,6313 & 1,6486 & 1,7012 & 1,6190 \\
\hline $\begin{array}{l}\text { Universitäts - } \\
\text { /College- } \\
\text { studierende }\end{array}$ & 1,8594 & 1,8881 & 1,9104 & 1,9908 & 1,9473 & 2,1311 & 1,7369 & 2,0552 & 1,9612 & 1,9648 & - \\
\hline $\begin{array}{l}\text { Krankenhaus- } \\
\text { betten }\end{array}$ & 0,4955 & 0,4825 & 0,4905 & 0,5413 & 0,5241 & 0,3269 & 0,3309 & 0,3537 & 0,3469 & - & 0,3826 \\
\hline Ärzte & 0,6311 & 0,5947 & 0,5762 & 0,5516 & 0,5398 & 0,3530 & 0,3466 & 0,3308 & 0,3389 & - & 0,3024 \\
\hline
\end{tabular}

Tab. 2: Das regionale Entwicklungsgefälle gemessen durch den gewogenen Variationskoeffizienten $(\mathrm{Vg})$ Regional development disparity based on a weighted variation coefficient $(\mathrm{Vg})$

Les disparités régionales de développement, mesurées en fonction du coefficient de variation pondéré (cv) Quellen: General Statistical Office 2001, 2000b, 2000c, 1999, 1997; Weltbank 1995; eigene Berechnungen

1. Der Anteil der Bevölkerung, der unterhalb der generellen Armutsgrenze lebte, sank insgesamt deutlich von $58,2 \%$ auf $37,4 \%$. Auch bei der Betrachtung der enger definierten Hungergrenze fiel der Prozentsatz von $24,9 \%$ auf $15 \%$ (zu den Armutskonzepten und -grenzen in Vietnam siehe Duong 2003: 88; Hemmer 1999: 136). Unter räumlichen Gesichtspunkten konnte die Armut in allen sieben Regionen - allerdings in unterschiedlichem Masse - abgebaut werden. Der Anteil der Bevölkerung unterhalb der oberen Armutsgrenze schwankte 1997/1998 auf regionaler Ebene zwischen 58,6\% im Nördlichen Bergland (Nordostund Nordwestregion) und 7,6\% in der Südostregion (General Statistical Office 2000a: 289).

2. Bei differenzierter Betrachtung der Veränderung der Armutssituation anhand der Entwicklung der Pro-Kopf-Konsumausgaben lassen die Daten auf eine Verschärfung des regionalen Entwicklungsgefälles schliessen. Erwartungsgemäss weist die bereits zum Zeitpunkt der ersten Befragung führende Südostregion - begünstigt von der wirtschaftlichen Dominanz des südlichen Entwicklungsdreiecks mit dem Zentrum HCMC - die mit Abstand höchsten Wachstumsraten auf, gefolgt vom Roten Fluss Delta mit der Metropole Ha Noi (Abb. 2). Insgesamt ist die Variationsbreite zwischen der «reichsten» und «ärmsten» Region im Untersuchungszeitraum von 1,9:1 auf 2,6:1 angestiegen.

3. Die Ergebnisse deuten ebenfalls auf ein sich verstärkendes Stadt-Land-Gefälle hin. In sechs von sieben Regionen sind die städtischen Konsumausgaben signifikant stärker gestiegen als die Ausgaben der ruralen Bevölkerung (WeLtBank 1999a: 71). Die höchsten Unterschiede weist die Nördliche Zentralküste auf. Ebenfalls überdurchschnittlich hoch sind die Disparitäten im Nördlichen Bergland, während das Rote Fluss Delta die einzige Region darstellt, in der die Steigerung der Konsumausgaben auf dem Land eine grössere Dynamik erreicht als in der Stadt. Auf nationaler Ebene vergrösserte sich das Verhältnis zwischen städtischen und ruralen Konsumausgaben pro Einwohner von $1,8: 1$ auf $2,2: 1$.

\section{Die Rolle des Staates im Entwicklungsprozess}

\subsection{Staatliche Entwicklungsplanung: Ausgleich versus Wachstum}

Die vietnamesische Regierung sieht sich bei der Erarbeitung ihrer Entwicklungsstrategien einem grundlegenden Dilemma ausgesetzt. Verkürzt lässt sich dieses als Zielkonflikt zwischen einer eher wachstumsorientierten und einer eher ausgleichsorientierten Raumwirtschaftspolitik umschreiben (REviLla Diez 1999: 108 ff.). Konkret geht es dabei um die Frage, ob die (in Entwicklungsländern in aller Regel) knappen Investitionsmittel räumlich konzentriert dort eingesetzt werden sollen, wo der kurzfristig zu erwartende gesamtwirtschaftliche Gewinn am höchsten ist, oder ob eine räumlich gleichmässige 


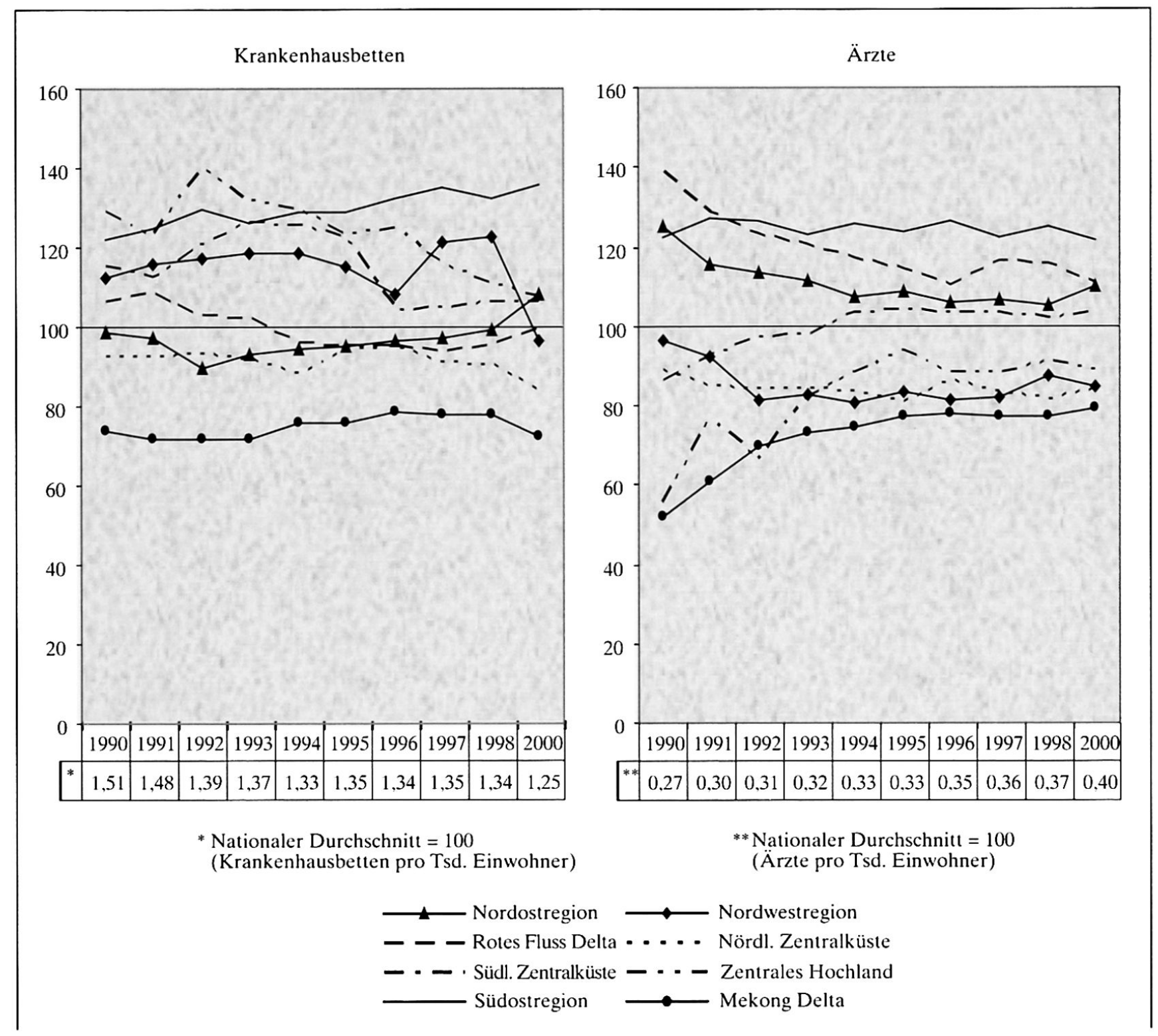

Abb. 1: Krankenhausbetten und Ärzte pro Kopf nach Regionen 1990-2000

Hospital beds and doctors per person according to regions 1990 to 2000

Nombre de lits d'hôpital et nombre de médecins/habitant, selon les régions (1990-2000)

Quellen: General Statistical Office 2001; eigene Berechnungen

Verteilung der Investitionen zum Zwecke einer ausgewogenen Entwicklung aller Landesteile angestrebt werden soll. Im ersteren Fall wird auf die Maximierung des BIP gesetzt. Hierzu wird die Entwicklung von wenigen sog. Growth Poles forciert, in der Annahme, dass in einer späteren Phase Entwicklungsimpulse von diesen Wachstumspolen auf die umliegenden Regionen ausgehen. Die ausgleichsorientierte Strategie nimmt hingegen eine Verlangsamung des gesamtwirtschaftlichen Wachstums in Kauf und zielt darauf, Investitionen in weniger entwickelte Regionen zu lenken. Im Vordergrund dieser
Strategie steht der Abbau von bestehenden regionalen Disparitäten.

Der vietnamesische Staat hat zu keiner Zeit des Reformkurses seine Führungsfunktion in Frage gestellt (TROGEMANN 1997: 141; Fahey 1997: 469). Als Folge ist er der wichtigste Akteur bei der Planung und Leitung von entwicklungs- und damit auch regionalpolitischen Massnahmen. Zielvorstellungen und Realität der Investitionsplanung, die in Vietnam als wichtiger Bestandteil der Regionalpolitik beurteilt wird (JACOBI 1996: 147), sollen deshalb einander gegenübergestellt werden. 


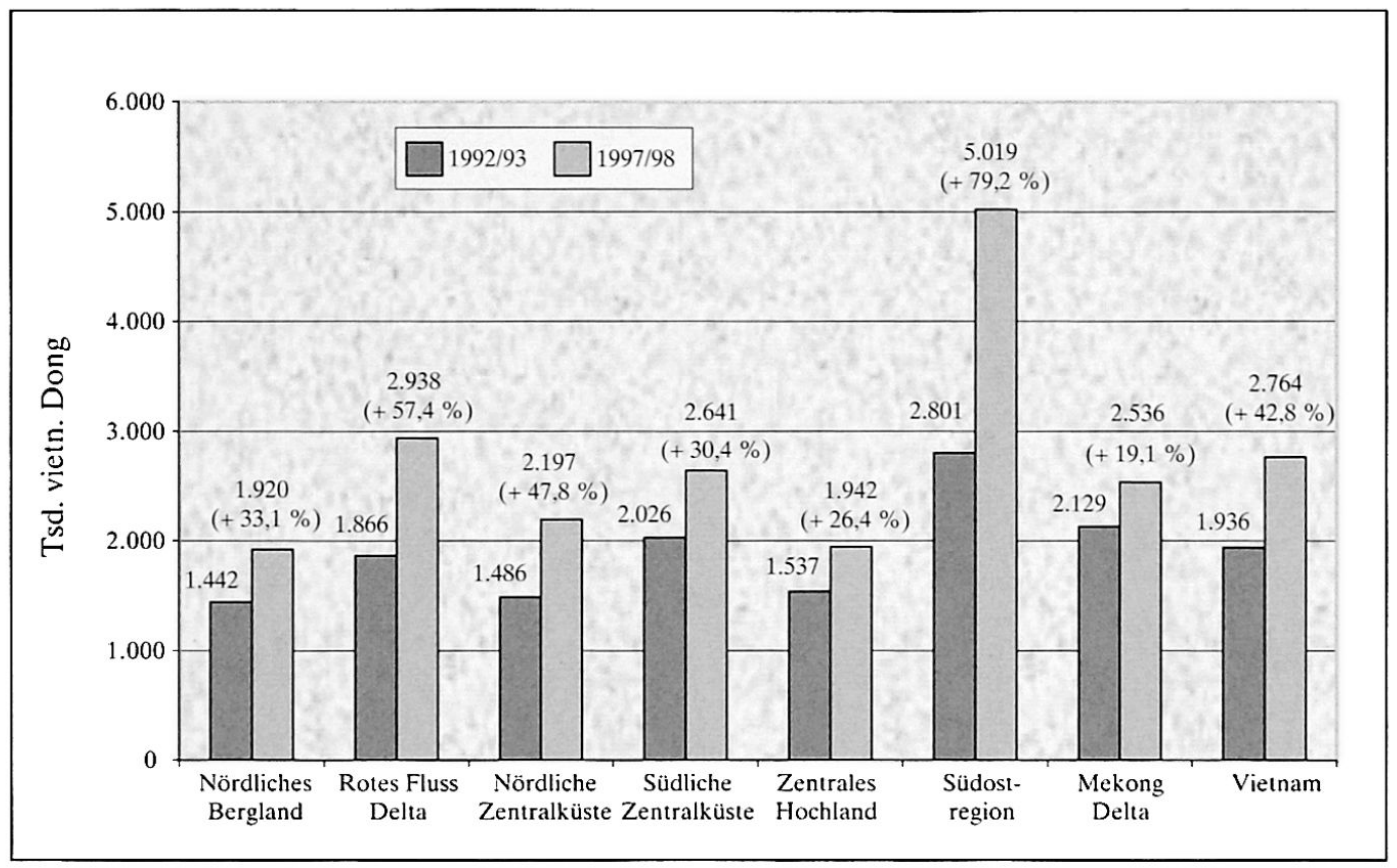

Abb. 2: Reale Pro-Kopf-Konsumausgaben nach Regionen 1992/93-1997/98

Actual consume expenditure per person according to regions 1992/93 to 1997/98

Dépenses réelles de consommation/habitant, selon les régions (1992/93-1997/98)

Quelle: General Statistical Office 2000a; Graphik: K.H. Duong

\subsection{Staatliche Investitionstätigkeit: Anspruch und Realität}

Grundlage für die Planung und Gestaltung von Investitionen des Staates sind in Vietnam die nationalen Entwicklungspläne. Für den Untersuchungszeitraum sind der 5. und 6. Fünfjahresplan (1991-1995 u. 1996-2000) sowie der Perspektivplan (1991-2000) relevant. Aus diesen Dokumenten lassen sich folgende Schlüsse hinsichtlich Planung und Wirklichkeit der staatlichen Investitionstätigkeit ziehen:

1. Bis Mitte der 1990er Jahre wurden die Überwindung der wirtschaftlichen Krisensituation und die Fortführung der Reformen als Übergang zum Marktmechanismus als prioritäre Aufgaben genannt. Dabei wurde von vornherein eine wachstumsorientierte Entwicklung angestrebt. Ein Konzept zur räumlichen Entwicklung im Sinne des Abbaus des regionalen Entwicklungsgefälles findet sich nirgends wieder. Lediglich sektoral sollte durch eine Neuausrichtung der Investitionsgestaltung die Entwicklung der Landwirtschaft verstärkt vorangetrieben werden. Tatsächlich aber sank der Anteil der staatlichen Investitionen in den Landwirtschaftsbereich zwischen 1990 und 1995 deutlich (Tab. 3). Stattdessen stand die Forcierung der industriellen Entwicklung im Vordergrund. Neben Verkehrsinfrastrukturprojekten lag das Hauptaugenmerk der Förderpolitik auf der Errichtung von
Exportförderzonen (EPZ) und Industrieparks (IP) (United Nations 1999: 13). Regional konzentrieren sich die EPZ und IP auf wenige Standorte in HCMC und Ha Noi sowie ihrer Umgebung. Die Bevorzugung der städtischen Wachstumszentren - der Anteil der staatlichen Infrastrukturausgaben für ländliche Regionen sank entsprechend zwischen 1991 und 1995 von 27,5\% auf 11,5\% (DANG Duc DAm 1999: 62) - verstärkte ihre ohnehin schon bestehenden Standortvorteile gegenüber den Peripherregionen.

2. Die Landwirtschaft als Haupteinkommensquelle für den Grossteil der Bevölkerung erhielt in der Investitionsplanung ab 1996 generell wieder besondere Beachtung bei der staatlichen Förderpolitik. Dennoch sollten die Investitionen in den Industriesektor die Fördermittel für den Agrarbereich um das 2,2- bis 2,4fache übersteigen, um dem Ziel der Industrialisierung zu entsprechen (DANG Duc DAM 1999: 64 ff.). Aber auch bei diesem Vorhaben offenbart die politische Praxis die Diskrepanz zwischen Planung und Wirklichkeit: Die Ausgaben beliefen sich zwischen 1996 und 1999 tatsächlich auf das 4- bis 5fache zugunsten des sekundären Sektors (Tab. 3). Die ohnehin geringen Investitionen in die Landwirtschaft wurden zudem ineffizient eingesetzt und kamen der ländlichen Bevölkerung kaum zugute. So floss der Löwenanteil der Investitions- 


\begin{tabular}{|l|c|c|c|c|c|}
\hline \multirow{2}{*}{ Jahr* } & \multirow{2}{*}{$\begin{array}{l}\text { Staatl. In- } \\
\text { vestitonen } \\
\text { insgesamt }\end{array}$} & \multicolumn{4}{|c|}{ davon } \\
\cline { 3 - 6 } & & \multicolumn{2}{|c|}{ Industrie und Bauwesen } & \multicolumn{2}{|c|}{ Land- und Forstwirtschaft } \\
\cline { 3 - 6 } & & absolut & $\%$ & absolut & $\%$ \\
\hline 1990 & $2.145,8$ & 824,2 & 38,4 & 367,0 & 17,1 \\
\hline 1991 & $2.383,0$ & $1.047,5$ & 44,0 & 372,9 & 15,6 \\
\hline 1992 & $3.333,2$ & $1.629,8$ & 48,9 & 422,1 & 12,7 \\
\hline 1993 & $5.898,4$ & $3.264,2$ & 55,3 & 522,6 & 8,9 \\
\hline 1994 & $5.729,8$ & $2.236,2$ & 39,0 & 536,8 & 9,4 \\
\hline 1995 & $5.559,8$ & $1.833,3$ & 33,0 & 641,7 & 11,5 \\
\hline 1996 & $35.894,4$ & $14.350,4$ & 40,0 & $3.044,0$ & 8,5 \\
\hline 1997 & $46.570,4$ & $14.687,1$ & 31,5 & $3.991,2$ & 8,6 \\
\hline 1998 & $52.536,1$ & $21.251,6$ & 40,5 & $5.116,8$ & 9,7 \\
\hline 1999 & $63.871,9$ & $25.411,3$ & 39,8 & $6.450,0$ & 10,1 \\
\hline
\end{tabular}

*) Angaben für 1990-1995 nach konstanten Preisen von 1989; Angaben für 1996-1999 nach laufenden Preisen

Tab. 3: Staatliche Investitionen 1990-1999 in Mrd. vietnamesischer Dong National investment between 1990 and 1999 in billion Vietnamese Dong Investissements étatiques en milliards de dong vietnamiens (1990-1999) Quellen: General Statistical OfFice 1997, 1999, 2000c, 2001

summen in die defizitären landwirtschaftlichen Staatsbetriebe, von denen kaum Beschäftigungseffekte ausgingen (WELTBANK 2000: 65).

Unter regionalpolitischen Gesichtspunkten erfuhr erst die Erarbeitung des 6. Fünfjahresplans einige wesentliche Neuerungen. Zur Gewährleistung eines Gesamtausgleichs wurden erstmalig konkrete Ausführungsmassnahmen als Grundlage für die Investitionsplanung formuliert (JACOBI 1996: 149). Zum Ausdruck kommt dies darin, dass eigens ein regionales Entwicklungsprogramm (programme of the development of territorial regions) zur Ausarbeitung kam. Daneben liessen weitere Massnahmen, wie das programme of the socio-economic development for mountainous areas, regionalpolitische Auswirkungen erwarten. In Zahlen ausgedrückt bedeutete dies die Absicht, $30 \%$ des Kapitals aus dem regionalen Haushalt auf die drei wirtschaftlichen Schwerpunktregionen - HCMC, Ha Noi sowie Da Nang/Quang Ngai in Mittelvietnam - und 70\% auf die restlichen Landesteile zu verteilen. Dieser Willenserklärung folgten in der Praxis aber kaum Änderungen gegenüber der bisherigen Regionalpolitik. Zwischen 1996 und 1998 flossen weiterhin über die Hälfte der Investitionsausgaben des Staates in die Südostregion und in das Rote Fluss Delta. Das bei weitem führende Wachstumszentrum HCMCDong Nai-Vung Tau konnte allein zwischen 1994 und 1998 28\% der staatlichen Investitionssumme auf sich vereinen (WeltBANK 1998: 19). Entsprechend gering war der Anteil, der den Weg in die restlichen Regionen des Landes fand.

\section{Resümee und Ausblick}

Die 1990er Jahre können zu Recht als die erfolgreichste Dekade in der Geschichte Vietnams bezeichnet werden. Dennoch offenbaren die Ergebnisse der Untersuchung, dass die regionalen Entwicklungsdisparitäten bei einer Reihe von Indikatoren nach wie vor extrem stark ausgeprägt sind. Zum Ende des Jahrtausends ist die Dominanz von HCMC und Ha Noi in vielen Lebensbereichen ungebrochen, während Da Nang als regionales Zentrum in Mittelvietnam vom Status eines Gegenpols zu den Wirtschaftsräumen im Norden und Süden des Landes noch weit entfernt ist.

Die in den letzten Jahren verstärkte Umsetzung von Massnahmen zum Abbau des regionalen Entwicklungsgefälles konnte bislang nur in Ansätzen zur gerechteren Verteilung der Wachstumserfolge beitragen. Erste Unruhen in einigen ländlichen Provinzen des Landes im Jahr 1997 verdeutlichen das gesellschaftliche Konfliktpotenzial einer solchen Entwicklung. Das zunehmende Entwicklungsgefälle zwischen Stadt und Land sowie Arm und Reich droht die politische Stabilität zu untergraben. Aus diesem Grund kann es sich Vietnam nicht leisten, die staatliche Entwicklungsförderung auf wenige Wachstumszentren zu konzentrieren und dabei so lange räumliche Ungleichgewichte zu tolerieren, bis durch den Wachstumsprozess genügend Investitionsmittel in diesen Zentren vorhanden sind, um Massnahmen zum Abbau der Disparitäten zu ergreifen. Die Notwendigkeit einer 
regional gerechten Verteilung der Wachstumserfolge ist essentielle Voraussetzung für die Gesamtentwicklung des Landes.

Vietnam steht zu Beginn des neuen Jahrtausends vor grossen Herausforderungen (vgl. UNITED NATIONS Development Programme 2001a; Ministry for PlanING AND INVESTMENT 2001). Ob das Land sich in den kommenden Jahren entwickeln kann, hängt nicht nur davon ab, ob die Wachstumserfolge der 1990er Jahre wiederholt werden können, sondern vor allem davon, ob und in welchem Masse es gelingt, einen wachsenden Anteil der Bevölkerung daran partizipieren zu lassen.

\section{Literatur}

Asian Development Bank (ed.) (2000): Key Indicators of Developing Asian and Pacific Countries 2000, Vol. 31. - Oxford, New York: Oxford University Press. BäNZIGER,A. (2000):Vietnams zweite Teilung. Während die Städte boomen, leben die Menschen auf dem Land weiter in Armut. - In: Süddeutsche Zeitung vom 30.11.2000: 10.

Bronger, D. \& M. Strelow (1996): Manila-BangkokSeoul. Regionalentwicklung und Raumwirtschaftspolitik in den Philippinen, Thailand und Südkorea. - = Mitteilungen des Instituts für Asienkunde 272, Hamburg: Selbstverlag.

Dang Duc Dam (1999): Volkswirtschaftliche Entwicklung und Unternehmensreformen in Vietnam Gegenwärtige Lage und Perspektive. - Ha Noi: The Gioi Verlag.

DuonG, K.H. (2003): Regionalentwicklung in Vietnam: Ausmaß und Dynamik des regionalen Entwicklungsgefälles in den 90er Jahren. - = Materialien zur Raumordnung 62, Geographisches Institut, RuhrUniversität Bochum.

FAHEY, S. (1997): Vietnam and the «third way»: The Nature of Socio-Economic Transition. - In: Tijdschrift voor Economische en Sociale Geografie 88, 5: 469-480.

General Statistical Office (GSO) (ed.) (2001): Statistical Yearbook 2000. - Ha Noi: Statistical Publishing House.

General Statistical Office (GSO) (ed.) (2000a): Vietnam Living Standards Survey 1997-1998. - Ha Noi: Statistical Publishing House.

General Statistical Office (GSO) (ed.) (2000b): Figures on Social Development in 1990s in Vietnam. Ha Noi: Statistical Publishing House.

General Statistical Office (GSO) (ed.) (2000c): Statistical Yearbook 1999. - Ha Noi: Statistical Publishing House.

General Statistical Office (GSO) (ed.) (1999): Socio-Economic Statistical Data of 61 Provinces and Cities in Vietnam. - Ha Noi: Statistical Publishing House.
General Statistical Office (GSO) (ed.) (1997): Statistical Yearbook 1996. - Ha Noi: Statistical Publishing House.

Gertler, P. \& J. Litvack (1998): Access to Health Care during Transition: The Role of the Private Sector in Vietnam. - In: Dollar, D., Glewwe, P. \& J. Litvack (eds): Household Welfare and Vietnam's Transition. = World Bank Regional and Sectoral Studies 1, Washington DC: $235-256$.

Hemmer, H.-R. (1999): Wirtschaftliche Systemtransformation und Armutsbekämpfung - eine ökonomische Analyse am Beispiel der Sozialistischen Republik Vietnam. - = Schriften des Zentrums für regionale Entwicklungsforschung der Justus-Liebig-Universität Gießen 78, Münster, Hamburg, London: LiT-Verlag. JACOBI, E. (1996): Planung in Vietnam: Woher Wohin. $-=$ Dissertation an der Hochschule für Wirtschafts-, Rechts- und Sozialwissenschaften, Universität St. Gallen, Nr. 1891, Bamberg.

Le Ba Thao (1997): Vietnam - The Country and its Geographical Regions. - Ha Noi: The Gioi Publishers. Ministry for Planing and Investment (MPI) (ed.) (2001):The 5-Year Plan for Socio-Economic Development (2001-05). - Draft Report, http://www.worldbank.org.vn/ strategy/cprs/pdf/5yr_Soc_Eco.pdf (26.06.2002).

Marr, R.L. (2002): Hanoi und Ho Chi Minh Stadt: Der Leidensweg zweier Städte. - In: SCHNEIDER-SLIWA, R. (Hrsg.): Städte im Umbruch. Die Neuorientierung von Berlin, Brüssel, Hanoi, Ho Chi Minh Stadt, Hongkong, Jerusalem, Johannesburg, Moskau, St. Petersburg, Sarajewo und Wien. - Berlin: Reimer-Verlag: 287-335.

NUSCHELER,F.(1999): Entwicklungssprünge, Disparitäten und Konfliktpotentiale im südostasiatischen Tigergehege. - In: KreIsel, W. (Hrsg.): Growing Gaps? Perspektiven für eine zukunftsfähige Regionalentwicklung im asiatisch-pazifischen Raum. - = Abera Conference Report 3, Hamburg: Abera-Verlag: 11-30.

Revilla Diez, J. (1999): Systemtransformation in der SR Vietnam - Regionalwirtschaftliche und soziale Auswirkungen. - In: Kreisel, W. (Hrsg.): Growing Gaps? Perspektiven für eine zukunftsfähige Regionalentwicklung im asiatisch-pazifischen Raum. - = Abera Conference Report 3, Hamburg: Abera-Verlag: 99-114.

Trogemann, G. (1997): Doi Moi - Vietnams Reformpolitik in der Retrospektive. $-=$ Passauer Beiträge zur Südostasienkunde 1, Lehrstuhl für Asienkunde, Universität Passau.

United Nations (UN) (ed.) (1999): Looking Ahead A United Nations Common Country Assessment of Vietnam. - http://www.undp.org.vn/projects/vie99002/ txgspeech.htm (19.05.2002).

United Nations Development Programme (UNDP) (ed.) (2001a): Summary of the socio-economic development strategy (2001-2010) and the Five-Year Socio-Economic Development Plan (2001-2005) for 
Vietnam. - Speech by Minister Tran Xuan Gia at the Roundtable Consultation on «From Strategy to Reality», http://www.undp.org.vn/projects/vie99002/ txgspeech.htm (23.05.2002).

United Nations Development Programme (UNDP) (ed.) (2001b): Key Economic Facts in Vietnam. http://www.undp.org.vn/undp/fact/stat/eng1/economics.pdf (20.06.2001).

WaIBEL, M. (2003): Die Altstadt von Hanoi: Ein Abbild urbaner Transformationsprozesse. - In: Geographische Rundschau 55, 1: 32-38.

Weltbank (ed.) (2000): Vietnam - Advancing Rural Development from Vision to Action. - Report prepared for the 1998 Consultative Group Meeting for Vietnam, December 14-15, 1999. - = Working Paper 18628 , Ha Noi.

WeLtBANK (ed.) (1999a):Vietnam Development Report 2000. Attacking Poverty. - Report prepared for the Consultative Group Meeting for Vietnam, December 14-15, 1999. - Ha Noi.

Weltbank (ed.) (1999b): Vietnam - Preparing for Take-off? How Vietnam can participate fully in the East Asian Recovery. - An Economic Report prepared for the Consultative Group Meeting for Vietnam, December 14-15, 1999. - = Working Paper 24472, Ha Noi.

WeLtBank (ed.) (1998): Vietnam: Rising to the Challenge. $-=$ Economic Report 18632, Ha Noi.

Weltbank (ed.) (1995): Vietnam - Economic Report on Industrialization and Industrial Policy. - = Economic Report 14645, Washington DC.

WeLtBank (ed.) (1994): Vietnam - Transforming a State owned Financial System. A Financial Sector Study of Vietnam. - Washington DC.

Wessel, K. (1998): Führende Tigerstaaten im asiatisch-pazifischen Raum. - In: Praxis Geographie 28, 9: 14-17.

WolfF, P. (1997): Vietnam - Die unvollendete Transformation. $-=$ Schriftenreihe des Deutschen Instituts für Entwicklungspolitik 113, Köln: Weltforum Verlag.

\section{Zusammenfassung: Vietnam - Regionale Dispari- täten in einem Entwicklungsland mit dynamischer Wirtschaftsentwicklung}

Vietnam konnte in den 1990er Jahren eindrucksvolle Wachstumserfolge erzielen. Im Beitrag wird gemäss dem Verständnis von «Entwicklung» als Partizipation der Bevölkerung am gesamtgesellschaftlichen Wohlstand folgenden Fragen nachgegangen: Wie ist die Entwicklung in den 1990er Jahren regional verlaufen? Haben alle Landesteile von der positiven gesamtnationalen Entwicklung profitieren können? Welche Rolle spielt der Staat im Entwicklungsprozess? Als Grundlage der Analyse von Ausmass und Dynamik des regionalen Entwicklungsgefälles dienen acht Indikatoren aus verschiedenen Lebensbereichen. Die Ergeb- nisse offenbaren gravierende regionale Disparitäten in nahezu allen Lebensbereichen. «Entwicklung» konzentriert sich demnach hauptsächlich in Ho Chi Minh City und $\mathrm{Ha}$ Noi bzw. ihren Umlandprovinzen. $\mathrm{Zu}$ diesem Ergebnis beigetragen hat die staatliche Entwicklungsplanung und -politik durch die anhaltende Bevorzugung der beiden Zentren des Landes - hier dargelegt am Beispiel der staatlichen Investitionstätigkeit. Insgesamt konnten im Untersuchungszeitraum die von der vietnamesischen Regierung erklärten Ziele zum Abbau der regionalen Disparitäten nicht erreicht werden.

\section{Summary: Vietnam - Regional disparity in a develop- ing country with dynamic economic development} In the 1990s, Vietnam boasted impressive economic growth rates. From the perspective of development being a reflection of the contribution of the population of a country towards general economic prosperity, the following issues are raised: Regional disparity of development in the 1990s, regional winners and losers, and the role of the state in the developmental process. Eight regionally relevant indicators served as a basis for the analysis of the extent and dynamics of regional disparity, revealing clear differences between regions. «Development» appears to be concentrated around the Greater Areas of Ho Chi Minh City and Ha Noi. It would seem that national development planning and policy play a role here, the continuing preferential treatment of these areas exemplified in this case by means of national investment. On the whole, the declaration of the Vietnamese government to combat regional disparity does not appear to have been fulfilled during the herein defined period of observation.

\section{Résumé: Le Vietnam - Disparités régionales dans un Etat en développement bénéficiant d'un développe- ment économique dynamique}

Durant les années 1990, le Vietnam a pu enregistrer des succès de croissance impressionnants. La présente étude pose les questions suivantes en interprétant le terme «développement» comme une participation de la population au bien-être sociétal général: De quelle façon le développement s'est-il déroulé, à l'échelle régionale, durant les années 1990 ? Toutes les parties du pays ont-elles pu bénéficier du développement national positif? Quel rôle joue l'Etat dans le processus de développement? La base de l'analyse de la dimension et de la dynamique du décalage du développement régional est constituée par huit indicateurs relevant de tous les domaines de l'existence. Selon cette démarche, le «développement» est concentré essentiellement à Ho Chi Minh Ville et à Hanoï, ainsi que dans leurs provinces environnantes. Ce résultat a été influencé par la politique et la planification étatiques mises en œuvre en matière de développement en vue de favoriser de manière permanente les deux centres du pays, 
une priorité qui est explicitée ici par l'exemple de l'investissement étatique. Dans l'ensemble toutefois, il n'a pas été possible, durant le laps de temps imparti à l'enquête, de vérifier dans quelle mesure les finalités affichées par le gouvernement vietnamien pour éliminer les disparités régionales ont pu être réalisées.

Dipl.-Geogr. Khanh Hung Duong, Geographisches Institut der Universität Basel, Abteilung Humangeographie / Stadt- und Regionalforschung, Klingelbergstrasse 27, CH-4056 Basel, Schweiz.

e-mail: Hung.Duong@unibas.ch

Manuskripteingang/received/manuscrit entré le 3.6.2004

Annahme zum Druck/accepted for publication/accepté pour l'impression: 6.6.2005 